\title{
SISTEMAS DE INTERFAZ NEURONAL Y SU DESARROLLO EN LAS NEUROCIENCIAS: REVISIÓN BIBLIOGRÁFICA SISTEMÁTICA ACERCA DE SU APLICACIÓN EN PERSONAS CON PARÁLISIS
}

\section{NEURAL INTERFACE SYSTEMS AND THE STUDY OF NEUROSCIENCE: A SYSTEMATIC REVIEW ON ITS APPLICATION IN PARALYZED PEOPLE}

\author{
Boris Lucero M. \\ Universidad Católica del Maule, Chile
}

María Teresa Muñoz-Quezada

Universidad Católica del Maule, Chile

\begin{abstract}
Resumen: El presente artículo tiene como objetivo sistematizar la evidencia de estudios de la última década sobre la aplicación de sistemas de interfaces neuronales en humanos con parálisis. Se utilizó una metodología de revisión bibliográfica sistemática considerando los términos "Neural interface system" y "Paralysis", tanto en idioma inglés como español en tres bases de datos (EbscoHost, PubMed y Scielo). Se encontraron 61 artículos, siendo incluidos 10 artículos en la revisión al aplicar los criterios de elegibilidad. Los resultados indican que esta es una área multidisciplinaria, con un enfoque basado en el paradigma de bucle cerrado, preferencia por interfaces invasivas y con objetivos que apuntan a tres campos de aplicación: Neuroprótesis, operación de computadores y aprovechamiento de hemisferio no dañado en personas con hemiplejia. Se concluye que el valor científico de estos estudios desde la neurotecnología, permiten proyectar a esta línea de investigación como una subdisciplina promisoria dentro del estudio de las neurociencias.
\end{abstract}

Palabras Clave: interfaz neuronal, parálisis, plasticidad neuronal, prótesis neuronal

\begin{abstract}
This article aims to synthesize reported evidence over the last decade on studies on the application of neural interface systems in humans with paralysis. A methodology of systematic literature review was used, considering the terms "Neural interface system" and "Paralysis" in both English and Spanish language in three databases (EbscoHost, PubMed and Scielo). 61 articles were found, and finally 10 articles met the eligibility criteria and were reviewed. The results indicate that this is a multidisciplinary field with an approach based on the closed-loop paradigm, with a preference for invasive interfaces and aimed at three main areas of application: Neuroprosthetics, operation of computers, and use of unaffected hemisphere in persons with hemiplegia. It is concluded that the scientific value of these studies from neurotechnology allows foreseeing this line of research as a promising sub-discipline within the study of neurosciences.
\end{abstract}

Keywords: neural interface, paralysis, neural plasticity, neural prosthetics

\section{Introducción}

La investigación para desarrollar sistemas que puedan ayudar a recuperar funciones de comunicación, sensoriales y de control en humanos que han sufrido lesiones o daños, ha concentrado el trabajo de una rama emergente de estudio en las neurociencias (Wolpaw, Birbaumer, McFarland, Pfurtscheller \& Vaughan, 2002). Esta agrupa a diversas sub-áreas que han recibido distintas denominaciones: interfaces cerebro-máquina (brain-machine interfaces, BMls), interfaz cerebro-computador (brain-com- puter interfaces, [BCls]), prótesis neuronales o sistemas de interfaz neuronal (Neural interface system, [NISs]).

La investigación en estos tópicos, ofrece la posibilidad de ayudar a las personas con discapacidades motoras y sensoriales severas para que tengan una mejor interacción con el mundo y una mejor calidad de vida (Dobkin, 2007). Sin embargo, mas allá de este importante aporte clínico, algunos neurocientíficos se preguntan si es que esta área de investigación constituye una disciplina científica dentro de las neurociencias, en cuanto que aporta algún conocimiento espe-

Correspondencia: Boris Lucero M. Facultad de Ciencias de la Salud. Universidad Católica del Maule, Chile.

Correo Electrónico: balucero@gmail.com 
cífico respecto al estudio del sistema nervioso humano, o más bien pertenecería a un sub-campo de la ingeniería biomédica tal como la ingeniería neuronal, la cual se enfoca principalmente en el desarrollo de tecnología útil (Chapin, 2000). Recientemente se ha observado un número creciente de publicaciones y presentaciones asociadas a esta área, en revistas neurocientíficas y conferencias (Hatsopoulos \& Donoghue, 2009).

A través de una revisión de la bibliografía de los estudios realizados en los últimos diez años en esta línea de investigación, el presente artículo pretende identificar los principales referentes de ésta área y los avances de la neurotecnología como una rama de la neurociencia, reflejados en los objetivos de estos estudios asociados al desarrollo de dispositivos que permitan el funcionamiento de prótesis en el ámbito de la intervención clínica en personas con parálisis.

\section{Antecedentes del desarrollo de interfaces cerebro-máquina}

Hans Berger fue quien descubrió el electroencefalograma (EEG) humano. En su publicación "Elektrenkephalogramm" del año 1929 (como se citó en Kugler, 1991), la que consistió en una primera revisión y comunicación de sus experimentos, se atrevió a especular en cuanto a la posibilidad de leer los pensamientos en base a los EEG usando sofisticados métodos de análisis matemático.

Por otra parte, Grey Walter, un brillante pionero del estudio de EEG, quien describió el contingente de variación negativa (CNV), comúnmente llamada "onda de expectancia" (una onda cerebral asociada con la atención y anticipación), construyó el primer analizador de frecuencia automático y el computador de "transientes promedio" con la intención de discriminar pensamiento o lenguaje encubierto en el EEG humano (Walter, 1964).

Otro referente fundacional se da en los años 60 , asociado a los orígenes de la neurofisiología conductual, siendo pioneros en este sentido los experimentos electrofisiológicos desarrollados por Evarts (1968) en el córtex motor primario (MI) de primates despiertos durante su actividad conductual. Sus hallazgos describieron cómo las tasas de disparo de neuronas individuales en MI de monos se correlacionaban significativamente con la fuerza o la torsión generada por las articulaciones durante el movimiento de los brazos.
En la misma época, Fetz (1969) publicó un artículo donde describió el condicionamiento operante de secuencias de potenciales de acción ("spike trains") corticales por medio de una técnica invasiva aplicada en animales. En este trabajo demostró que un mono podía tener control volitivo de la actividad de neuronas únicas en Ml usando biofeedback visual y recompensa. A los monos se les entregaba refuerzo cuando lograban mover la aguja de un medidor análogo cuya posición era controlada por la tasa de disparo de una neurona que estaba siendo registrada. Este experimento entregó evidencia inicial en cuanto a que los primates podían aprender control por medio de retroalimentación de la actividad neuronal sin la intervención de movimientos.

Otro hito importante corresponde a un artículo clásico publicado por Humphrey, Schmidt, y Thompson (1970), quienes demostraron que el curso de tiempo del movimiento de una articulación de la muñeca de un mono podía ser predicho con bastante precisión usando una pequeña población de neuronas MI registradas simultáneamente, explicando además el tipo de población o procesamiento de ensamble que ocurre en el córtex para producir movimiento.

\section{Paradigmas de investigación en sistema de interfaces neuronales}

Los dos estudios mencionados en la sección anterior (Fetz, 1969; Humphrey et al., 1970), dan lugar a dos tradiciones o modalidades de investigación de las interfaces neuronales:

a) De control de bucle cerrado (closedloop control), del cual el estudio de Fetz es un ejemplo paradigmático. En estudios recientes encontramos un enfoque en esta línea en el trabajo de Velliste, Perel, Spalding, Whitford, y Schwartz (2008), en el cual se demostró que un mono (macaca mulatta) podía controlar la posición tridimensional del efector de término de un robot, como también su pinza unidimensional para alimentarse a sí mismo. La meta del animal era controlar al robot, no su propio brazo, para agarrar la comida y llevarla hasta su boca, lo cual es lo característico en esta primera modalidad de estudio de interfaces neuronales.

b) De predicción de bucle abierto (open-loop prediction) o que decodifican desde registros en múltiples sitios, siendo un ejemplo de esto el estudio mencionado previamente de Humphrey et al. (1970). Un trabajo más reciente que se enmarca en este tipo de enfoque es el de 
Wessberg et al. (2000), donde describieron que era posible predecir en tiempo real la posición bidimensional y tridimensional de la mano de un mono usando ensambles neuronales desde varias áreas corticales. Esta señal de predicción fue usada luego para mover un brazo robótico en una ubicación remota, demostrando que había información suficiente presente en la muestra de neuronas como para reconstruir movimientos del brazo y que este movimiento podía ser ejecutado casi en tiempo real. Este caso corresponde a una predicción de bucle abierto, ya que el animal no tenia noción alguna de estar moviendo el brazo robótico, no recibiendo ningún tipo de retroalimentación sensorial desde el robot, no correspondiendo por lo tanto, a un estudio de control de bucle cerrado.

Algunos han argumentado que los estudios de predicción de bucle abierto serían solo parte del paradigma de electrofisiología conductual y no de interfaces neuronales, sin embargo los estudios de esta modalidad de decodificación fueron esenciales en la formación de las bases para los futuros avances en interfaces neuronales, siendo especialmente importantes las contribuciones de Georgopoulos, Kalaska, Caminiti, y Massey (1982); y Georgopoulos, Kettner, y Schwartz (1988), quienes demostraron como poblaciones de neuronas activadas ("spiking neurons") podían predecir la cinemática de un brazo en el espacio. Estos estudios son un ejemplo de cómo trabajos que no estuvieron inspirados en la idea de construir interfaces neuronales entregaron el avance de conocimiento necesario para desarrollarlas. También se podría agregar que entregaron conocimiento de base respecto al funcionamiento neuronal y por tanto realizaron una contribución fundamental al conocimiento neurocientífico.

Por otra parte, en cuanto al desarrollo mismo de las interfaces neuronales han existido también dos enfoques alternativos, las interfaces invasivas y las interfaces no invasivas, que aun cuando comparten como raíz el ser estudios experimentales con animales, se originan desde diferentes tradiciones (Birbaumer, 2006).

Las interfaces invasivas, consisten en rejillas de electrodos que han sido implantadas ya sea en la corteza motora de pacientes paralizados (Donoghue, 2008; Donoghue et al., 2007a; Donoghue, Nurmikko, Black, \& Hochberg, 2007b; Hochberg et al., 2012), la corteza premotora de monos (Carmena et al., 2003), o áreas motoras parietales (Schwartz, Taylor, \&
Tillery, 2001). Lo que se busca es reconstruir movimientos intencionales desde patrones de disparo neuronal. Basados en enfoques de codificación aislada ("sparse coding") respecto al aprendizaje motor y de vectores de codificación direccional de neuronas motoras, es posible reconstruir movimientos complejos a partir de pocas neuronas motoras utilizando algoritmos simples. De hecho algunos grupos de trabajo (Carmena et al., 2003) han demostrado en estudios con monos que luego de un extenso entrenamiento de un movimiento de alcanzar y agarrar, el patrón de disparo de apenas 32 neuronas ya es suficiente para ejecutar dicho movimiento directamente con un brazo artificial.

A su vez en la metodología no invasiva el input es registrado desde el cuero cabelludo por medio de EEG. Estos pueden ser categorizados en los que utilizan inputs evocados o inputs espontáneos (Wolpaw et al., 2002). Los inputs evocados se obtienen por la estimulación sensorial estereotipada provista por la interfaz (e.g. EEG producido por letras intermitentes ["flashing letters"]). A su vez, los inputs espontáneos no dependen para su generación de estímulo alguno (e.g. ritmos del EEG en la corteza sensorio-motora). Para la adquisición de la señal, el input escogido es adquirido por el registro de los electrodos, amplificado y digitalizado. Ejemplos de este tipo de enfoque no invasivo se pueden encontrar en Kübler et al. (2005) y en Nijboer et al. (2008).

\section{Aplicación de sistema de interfaz neuronal en personas con parálisis}

La parálisis consiste en una limitación del movimiento normal producto de la desconexión de señales motoras desde los músculos. En el peor de los casos se manifiesta como "síndrome de cautiverio" en el cual existe una parálisis completa, bloqueando además toda forma de comunicación. Su origen se da de diferentes modos: por lesión en las vías motoras descendientes de la médula espinal, el tallo cerebral, o cerebelo debido a infarto o trauma; trastornos degenerativos que llevan a la pérdida de neuronas motoras (como es el caso en la esclerosis lateral amiotrófica) o de las vías motoras (e.g. en la esclerosis múltiple); trastornos degenerativos a nivel del músculo (distrofia muscular); o por pérdida de extremidades (Donoghue et al., 2007b).

Basado en los conceptos actuales de plasticidad y respuesta a lesiones, los humanos que sufren una parálisis severa, como es el caso 
de la tetraplejia(pérdida de movimiento en los 4 miembros), independientemente de su causa, pierden el potencial de controlar la actividad neuronal o incluso la actividad neuronal funcional luego de que las áreas motoras se han desconectado del cuerpo (Enzinger et al., 2008). No obstante desde hace más de una década existe evidencia (Kennedy \& Bakay, 1998; DiLorenzo, Walker, Davis, \& Gross, 2006) en cuanto a que una persona con parálisis severa podría generar activación neuronal de modo intencional. Algunos estudios clínicos luego incluso han demostrado que años después de una lesión en la medula espinal (Spinal cord injury, [SCI]) o de un accidente cerebro vascular (ACV) en el tallo encefálico, la activación de espigas en MI como también de actividad de potencial de campo local (local field potential, [LFP]) persiste y ambas señales pueden ser moduladas inmediatamente de forma intencional o por intentos de movimiento (Hochberg et al., 2006; Truccolo, Friehs, Donoghue, \& Hochberg, 2008). Todos estos hallazgos son llamativos en primer lugar en cuanto a que $\mathrm{SCl}$ daña los axones de $\mathrm{MI}$, lo cual podría resultar en inactividad o pérdida celular. En segundo lugar, la plasticidad después de la falta de uso o lesión se esperaría que produjera una marcada reestructuración del córtex, tal vez debido a que otras áreas tomarían el lugar de las previas (Donoghue, Suner, \& Sanes, 1990). En los estudios desarrollados por Donoghue (2008), se observó que personas con tetraplejia producto de diversas causas $(\mathrm{SCl}, \mathrm{ACV}$ pontino, esclerosis lateral amiotrófica [Amyotrophic Lateral Sclerosis, ALS]) fueron capaces de generar actividad en MI inmediatamente luego de pedirles que imaginaran acciones del brazo. Aunque aún es necesario confirmar la posible generalización de esos hallazgos, es prometedor que el sustrato neural para proveer el control de una interfaz se haya obtenido.

Además de lo anterior, cabe destacar que tanto los potenciales de campo como las señales de espiga, las cuales resultan útiles para la implementación de sistema de interfaces neuronales, persistan largo tiempo luego del surgimiento de la parálisis por lesiones o enfermedades neurodegenerativas (Kim, Simeral, Hochberg, Donoghue, \& Black, 2008). Esto sugeriría que una gran población de individuos con discapacidades asociadas a parálisis podrían beneficiarse de la tecnología de interfaz neuronal. A pesar de ser necesario un mayor desarrollo del área de estudio para que las personas con tetraplejia al- cancen movimientos rápidos y con total habilidad bajo su control neuronal, en el momento actual, para las personas que no tienen o poseen un muy limitado rango de movimientos voluntarios de su propias extremidades, incluso las acciones más básicas de alcance y agarre, podrían implicar el recuperar la habilidad de comer y beber autónomamente, mejorando invaluablemente su calidad de vida.

Si bien hasta ahora las demostraciones en humanos de la potencial efectividad de estas interfaces han sido limitadas en cuanto a número, resultaría útil revisar los estudios recientes que se han desarrollado en esta área de modo tal de entender desde qué disciplinas se aborda esta línea de conocimiento y de manera importante, cuáles son las principales líneas de trabajo y proyección en el estudio del aprovechamiento de las capacidades que conservan las personas con parálisis, a través de la aplicación y uso de interfaces neuronales.

Frente a la problemática anteriormente mencionada surgen entonces las siguientes preguntas: ¿Cuáles son las disciplinas de origen de los estudios en ésta área? ¿Qué paradigma es utilizado preferentemente en estos estudios (bucle abierto o bucle cerrado)? ¿Qué tipo de interfaz se utiliza (invasiva o no invasiva)? ¿Qué tipo de parálisis consideran? ¿Cuáles son los objetivos de estos estudios?

El presente trabajo entonces tiene como objetivo el sistematizar la evidencia de los estudios que han investigado la aplicación de sistemas de interfaces neuronales en humanos con parálisis y sintetizar sus características en cuanto a paradigma del enfoque, interfaz utilizada, tipo de parálisis y objetivo de estudio.

\section{Metodología}

Se realizó una revisión sistemática de literatura científica en las bases de datos electrónicas EBSCOhost, Pub-Med y SciELO. Para la búsqueda se utilizaron como términos claves "Neural Interface System" y "Paralysis", tanto en el idioma inglés como español. Como criterios de elegibilidad para los artículos se consideró el que hubieran sido publicados en los últimos 10 años, refirieran solo investigación en sujetos humanos, que correspondieran a publicaciones científicas indizadas y arbitradas y que abordaran directamente el estudio de la interfaz cerebro-máquina aplicada en personas con parálisis de origen neurológico. Aplicando los términos de búsque- 
da se identificaron un total de 61 artículos de los cuales, una vez eliminados los duplicados, se revisaron 55 , siendo seleccionados 31 que cumplían con los criterios de elegibilidad para revisión de texto completo. Una vez revisados, finalmente 10 artículos fueron parte de la síntesis, debido a que abordaban específicamente en sus reportes de investigación el desarrollo de sistemas de interfaces neuronales y su aplicación en personas con parálisis de origen neurológico (ver Figura 1). De cada uno de estos estudios se extrajo información referente a la disciplina de origen de los autores principales, paradigma del estudio (bucle abierto o bucle cerrado), tipo de interfaz utilizada (no invasiva o invasiva), tipo de parálisis de los participantes y objetivo principal considerado.

\section{Resultados}

Los resultados obtenidos en base a los 10 trabajos extraídos por medio de la revisión sistemática bibliográfica se muestran en detalle en la Tabla 1, organizados de acuerdo a los parámetros descritos previamente. A continuación se expondrán los resultados obtenidos para las distintas características previstas de acuerdo al objetivo planteado en este trabajo.

Al indagar acerca de las disciplinas de origen de los autores principales, se observa que los estudios son realizados por diferentes disciplinas y especialidades, siendo la mayor cantidad de contribuciones desde el área de la Ingeniería $(n=4)$, correspondiendo la mitad específicamente a la rama de la ingeniería biomédica.
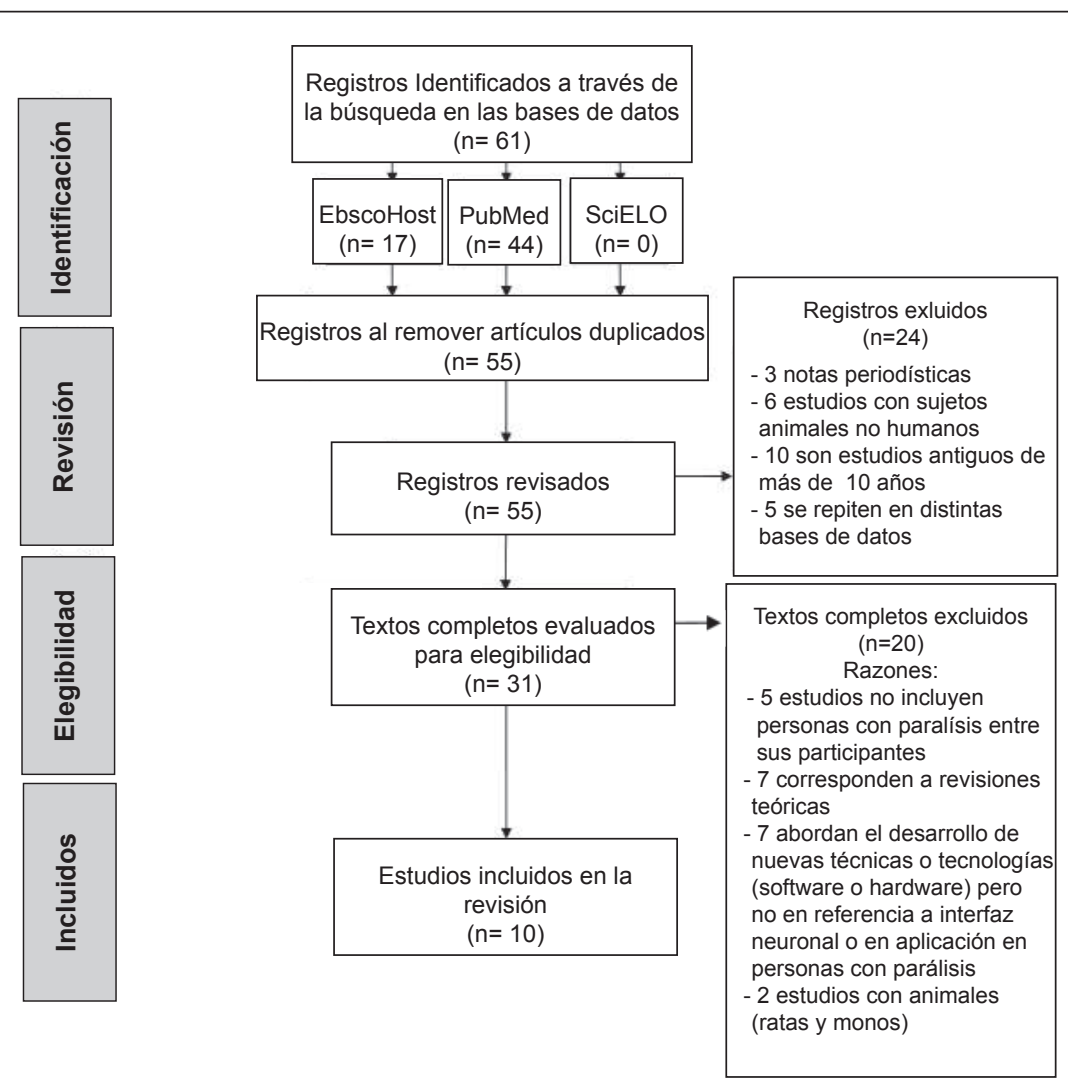

Figura 1. Diagrama de flujo que sintetiza la revisión sistemática bibliográfica realizada y sus resultados. Adaptado de la "Declaración PRISMA: una propuesta para mejorar la publicación de revisiones sistemáticas y metaanálisis", por G. Urrutia \& X. Bonfill, 2010, Medicina clínica, 135(11), p. 507-511. 
Tabla 1. Resumen de los estudios y sus principales características en cuanto a disciplina de origen de los autores, paradigma de base, tipo de interfaz, tipo de parálisis y sintesis del objetivo del estudio

\begin{tabular}{|c|c|c|c|c|c|}
\hline $\begin{array}{l}\text { Autor, año y } \\
\text { país }\end{array}$ & $\begin{array}{l}\text { Disciplina de } \\
\text { origen }^{a}\end{array}$ & $\begin{array}{l}\text { Paradigma de } \\
\text { estudio } \\
\text { (Bucle abierto } \\
\text { - cerrado) }\end{array}$ & $\begin{array}{l}\text { Tipo de } \\
\text { interfaz } \\
\text { (Invasiva/No } \\
\text { invasiva) }\end{array}$ & $\begin{array}{l}\text { Tipo de } \\
\text { Parálisis }\end{array}$ & Objetivo \\
\hline $\begin{array}{l}\text { Bundy et al., } \\
\text { 2012; EE.UU. }\end{array}$ & $\begin{array}{l}\text { Ingeniería } \\
\text { Biomédica }\end{array}$ & Bucle cerrado & No invasiva & Hemiplejia & $\begin{array}{l}\text { Evaluar uso de actividad motora ipsilateral del } \\
\text { hemisferio cerebral no dañado para control de } \\
\mathrm{BCl} \text { en pacientes con hemiplejia posterior a } \\
\text { infarto cerebral }\end{array}$ \\
\hline $\begin{array}{l}\text { Caria et al., } \\
\text { 2011; Alemania }\end{array}$ & $\begin{array}{l}\text { Psicología médica } \\
\text { y Neurobiología } \\
\text { conductual }\end{array}$ & Bucle cerrado & No invasiva & Hemiplejia & $\begin{array}{l}\text { Evaluación con neuroimágenes de la reorga- } \\
\text { nización cerebral de pacientes con hemiplejia } \\
\text { posterior a infarto cerebral luego de recibir en- } \\
\text { trenamiento en } \mathrm{BCl} \text { y fisioterapia }\end{array}$ \\
\hline $\begin{array}{l}\text { Chadwick et al., } \\
\text { 2011; EE.UU }\end{array}$ & $\begin{array}{l}\text { Ingeniería } \\
\text { Biomédica }\end{array}$ & Bucle cerrado & Invasiva & Tetraplejia & $\begin{array}{l}\text { Evaluar la viabilidad del uso de una tecnología de } \\
\text { simulación computacional de un brazo paralizado } \\
\text { reanimado con sus características dinámicas, } \\
\text { para la restauración de la función del miembro } \\
\text { superior en personas con tetraplejia, antes de } \\
\text { implementar efectivamente un sistema de esti- } \\
\text { mulación eléctrica funcional(FES) a través de la } \\
\text { implantación de un dispositivo cortical }\end{array}$ \\
\hline $\begin{array}{l}\text { Hochberg et al., } \\
\text { 2012; EE.UU }\end{array}$ & Neurología & $\begin{array}{l}\text { Bucle abierto } \\
\text { Bucle cerrado }\end{array}$ & Invasiva & Tetraplejia & $\begin{array}{l}\text { Demostrar que personas que no tienen control } \\
\text { funcional del brazo debido a parálisis podian } \\
\text { utilizar la actividad generada por el ensamble neu- } \\
\text { ronal que realiza movimiento intencional del brazo } \\
\text { y la mano para alcanzar y agarrar objetos con } \\
\text { un brazo robótico en un espacio tridimensional }\end{array}$ \\
\hline $\begin{array}{l}\text { Hochberg et al., } \\
\text { 2006; EE.UU }\end{array}$ & Neurología & $\begin{array}{l}\text { Bucle abierto }{ }^{b} \\
\text { Bucle cerrado }\end{array}$ & Invasiva & Tetraplejia & $\begin{array}{l}\text { Ensayo clínico piloto para obtener evidencia } \\
\text { inicial respecto a que un humano con parálisis } \\
\text { podía operar una prótesis neuromotora usando } \\
\text { actividad de ensambles neuronales de } \mathrm{MI} \text { como } \\
\text { fuente de control }\end{array}$ \\
\hline $\begin{array}{l}\text { Kim et al., 2008; } \\
\text { EE.UU }\end{array}$ & $\begin{array}{l}\text { Ciencias } \\
\text { computacionales }\end{array}$ & $\begin{array}{l}\text { Bucle abierto } \\
\text { Bucle cerrado }\end{array}$ & Invasiva & Tetraplejia & $\begin{array}{l}\text { Comparar el desempeño en personas con pará- } \\
\text { lisis del control neuronal de un cursor aplicando } \\
\text { dos enfoques y algoritmos de decodificación } \\
\text { basados en el control de la posición o velocidad } \\
\text { del cursor }\end{array}$ \\
\hline $\begin{array}{l}\text { Kim et al., } \\
\text { 2011;EE.UU }\end{array}$ & $\begin{array}{l}\text { Ciencias } \\
\text { computacionales }\end{array}$ & Bucle cerrado & Invasiva & Tetraplejia & $\begin{array}{l}\text { Permitir a personas con parálisis la operación } \\
\text { continua del puntero y el click en un computador } \\
\text { usando un sistema de interfaz neuronal equiva- } \\
\text { lente a un mouse de computador }\end{array}$ \\
\hline $\begin{array}{l}\text { Perge et al., } \\
\text { 2013; EE.UU }\end{array}$ & Ingeniería & Bucle cerrado & Invasiva & Tetraplejia & $\begin{array}{l}\text { Evaluar en un ensayo clínico piloto con personas } \\
\text { con parálisis la naturaleza y extensión de la } \\
\text { inestabilidad en el disparo de señales de pobla- } \\
\text { ciones neuronales, a partir de sus amplitudes y el } \\
\text { impacto que tienen en la respuesta decodificada } \\
\text { (control de un cursor) }\end{array}$ \\
\hline $\begin{array}{l}\text { Piccione et al., } \\
\text { 2006; italia }\end{array}$ & Neurofisiología & Bucle cerrado & No invasivo & Tetraplejia & $\begin{array}{l}\text { Describir el uso tanto por parte de personas sanas } \\
\text { como de pacientes con tetraplejia del potencial } \\
\text { evocado de la onda P } 300 \text { como una señal de } \\
\text { control para una interfaz cerebro-computador }\end{array}$ \\
\hline $\begin{array}{l}\text { Simeral et al., } \\
\text { 2011; EE.UU }\end{array}$ & Ingeniería & Bucle cerrado & Invasivo & Tetraplejia & $\begin{array}{l}\text { Examinar el control del puntero y el click alcanza- } \\
\text { do por un individuo con tetraplejia luego de cinco } \\
\text { días consecutivos con ocasión de haber pasado } \\
\text { los } 1000 \text { días desde que se le implantó en su } \\
\text { corteza una matriz de electrodos fabricados de } \\
\text { semiconductores de silicona }\end{array}$ \\
\hline
\end{tabular}

a Refiere la disciplina de origen del autor principal

$b$ Observado experimentalmente demostrando control neuronal de la tarea por medio de acciones intencionales/imaginadas (no debido a la mínima capacidad de movimiento de cabeza, cuello u hombro que mantenía el participante en el experimento)

c Aplicado solo en la fase de entrenamiento del experimento

$\mathrm{BCl}=$ Brain-computer interface (interfaz cerebro-computador); $\mathrm{Ml}=$ corteza motora primaria 
Luego le siguen las ciencias computacionales y la neurología con dos contribuciones cada una. Finalmente la Neurofisiología y la psicología médica/neurobiología conductual se observan con una contribución cada una.

El paradigma de bucle cerrado fue utilizado en todos los estudios que fueron parte de la revisión. La utilización del paradigma de bucle abierto fue declarada de manera complementaria únicamente en tres estudios, siendo reportado en un caso como una observación experimental (Hochberg et al., 2006) y en otros para efectos de entrenamiento experimental (Kim, Simeral, Hochberg, Donoghue, \& Black, 2008) o de calibración para el uso de la interfaz neuronal (Hochberg et al., 2012).

En cuanto a las características de la interfaz utilizada, siete de los estudios utilizaron una de tipo invasivo consistente en una matriz de silicona de 4x4 milímetros que contiene 100 micro-electrodos, la cual va implantada en la superficie de la corteza, para el registro directo de potenciales de acción y potenciales de campo local en el cerebro, dentro de los ensayos clínicos del denominado proyecto Braingate (Donoghue, 2008). Los tres estudios restantes utilizaron interfaces no invasivas, siendo el EEG la técnica escogida (Bundy et al., 2012; Caria et al., 2011; Piccione et al., 2006).

Respecto al tipo de parálisis de los participantes en los estudios, mayoritariamente correspondían a personas con tetraplejia, siendo incluidos en ocho de los reportes, mientras que en sólo dos estudios se consideraron pacientes con hemiplejia posterior a infarto cerebrovascular (Bundy et al., 2012; Caria et al., 2011).

Finalmente, se puede distinguir que los distintos objetivos de los estudios revisados se pueden agrupar en líneas comunes de investigación, pudiendo ser descritas tres áreas principales de desarrollo en el tópico de interfaces neuronales en personas con parálisis, que son las que se detallan a continuación:

a) Estudios respecto a operación neural de prótesis de extremidad superior: Tres de los estudios revisados (Chadwick et al., 2011; Hochberg et al., 2012; Hochberg et al., 2006), tienen en común el estar dirigidos a obtener evidencia que permita demostrar que, personas con parálisis pueden operar una prótesis de una extremidad superior por medio de ensambles neuronales de MI como fuente de control. Los resultados de estos tres estudios apuntan en la misma dirección, entregando evidencia de que personas con tetraplejia fueron capaces de controlar multidimensionalmente de forma directa y útil dispositivos complejos (un brazo robótico), a partir de pequeñas muestras de señales neuronales registradas por medio del implante cortical, llegando incluso a movimientos más específicos de la extremidad robótica, como los de alcance y agarre (Hochberg et al., 2012).

b) Estudios respecto a operación neural de puntero y click de mouse: Cinco de los estudios incluían como objetivo el obtener evidencia para probar o perfeccionar una tecnología consistente en el uso de puntero y click de mouse para la operación de un computador, por medio del control surgido desde las señales de ensambles neuronales en personas con parálisis (Kim et al., 2008; Kim et al., 2011; Perge et al., 2013; Piccione et al., 2006; Simeral, Kim, Black, Donoghue, \& Hochberg, 2011).

Cuatro de los estudios obtuvieron sus datos usando una interfaz invasiva. Los resultados de uno de ellos (Perge et al., 2013), evidenciaron que la adquisición de señales y los métodos de decodificación que se pueden adaptar a los reportes de inestabilidades, podría mejorar el desempeño de las interfaces intracorticales, demostrado experimentalmente por medio del manejo de un cursor de computador. Los otros tres estudios obtuvieron resultados que apoyaban la viabilidad del uso de señales de un pequeño ensamble de neuronas de la corteza motora de personas con tetraplejia para controlar el puntero y el cliqueo de un cursor en el espacio bidimensional de un computador personal (Kim et al., 2008; Kim et al., 2011), incluso después de 1000 días de la implantación del sensor intracortical (Simeral et al., 2011).

Por su parte, el estudio que utilizó una interfaz no invasiva por medio de potenciales evocados (P300) usando EEG (Piccione et al., 2006), no obtuvo resultados tan concluyentes en cuanto a respaldar su efectividad, reportando que el sistema necesitaba de un período de entrenamiento previo para completar de la tarea y el logro de un buen desempeño. Además, mostró un mejor funcionamiento en personas sanas que en personas con parálisis.

c) Estudios en pacientes con hemiplejia: Dos de los estudios se enfocaron en estudiar la situación de pacientes con parálisis en la mitad de su cuerpo, como consecuencia de las secue- 
las de un infarto cerebro vascular, usando una interfaz no invasiva, buscando evaluar el posible aprovechamiento del hemisferio no dañado para la operación de interfaces neuronales (Bundy et al., 2012) y comprender cómo se reorganiza su cerebro luego de ser entrenados en el uso de interfaces cerebro-computador (Caria et al., 2011).

Uno de los estudios (Bundy et al., 2012) logró demostrar por primera vez que las señales de EEG del hemisferio cerebral no afectado se asociaban con movimiento explícito e imaginado de la mano afectada, demostrando la viabilidad de que los sobrevivientes de infarto cerebral puedan controlar el cursor de un computador de forma unidimensional de manera rápida y precisa. El otro estudio (Caria et al., 2011) revisó neuroimágenes de pacientes con hemiplejia demostrando que, aún cuando no era posible discernir los efectos individuales y específicos del uso de $\mathrm{BCl}$ respecto a los diferentes métodos de rehabilitación utilizados, si se observaba una sustancial y clínicamente significativa recuperación de la función motora de la mano a través del tiempo luego del uso de una interfaz cerebro-computador no invasiva (entrenamiento en el control de una órtesis acoplada a su mano por medio de la modulación de ondas cerebrales registradas en EEG).

\section{Discusión}

A partir de la revisión realizada, queda en evidencia que el estudio de los sistemas de interfaz neuronal comprende un área de trabajo de encuentro de distintas líneas del conocimiento, donde se requiere un esfuerzo multidisciplinario, siendo los mayores aportes liderados desde la Ingeniería, contemplando esfuerzos investigativos adicionales desde áreas complementarias que van desde la neurología, hasta áreas en apariencia tan disímiles como las ciencias computacionales y la psicología médica.

El uso mayoritario del paradigma de bucle cerrado en las investigaciones reportadas en los artículos revisados, tiene directa relación con las características de los experimentos desarrollados, incluyéndolo explícitamente dentro de lo que se planifica para la decodificación de las señales neuronales, ya que su característica principal es que permite que la retroalimentación del sistema controle y regule la respuesta configurando con esto un sistema dinámico, en el cual se realizan adaptaciones ante las perturbaciones de sus señales de entrada. Esto en el contexto del control neural de dispositivos externos, ya sea neuro-prótesis o computadores, resultaría deseable ya que posibilitaría el que el sistema funcione de manera adaptativa, y por lo tanto, más efectiva.

Se debe hacer notar que el $70 \%$ de los artículos revisados, son parte de un mismo proyecto de ensayos clínicos con personas con parálisis denominado Braingate (Donoghue et al., 2007b), a partir del cual se ha creado un sistema de interfaz neuronal compuesto por un sensor (el implante cortical), un decodificador (un conjunto de hardware y software computacional que transforma las señales del cerebro en comandos para manejar un dispositivo externo) y un dispositivo externo, ya sea de comunicación o una extremidad robótica (Lu, Patil, \& Chestek, 2012).

Dentro de este grupo de estudios está por ejemplo, el caso del trabajo desarrollado por Hochberg et al. (2012), quienes demostraron que dos personas que llevaban largo tiempo con la condición de tetraplejia podían ser capaces de utilizar un sistema de interfaz basado en el control de un brazo robótico para ejecutar movimientos tridimensionales de alcance y agarre. Los participantes controlaron el brazo y la mano en un espacio amplio sin entrenamiento explicito, usando señales decodificadas de una población pequeña de neuronas de la corteza motora (MI) registrada con una rejilla de microelectrodos de 96 canales. Uno de los participantes en el estudio, quien había recibido el implante del sensor 5 años antes, también utilizó el brazo robótico para tomar café desde una botella. Aunque los movimientos de alcance y agarre no fueron igual de rápidos o precisos que los de una persona capaz de ocupar su propio cuerpo, los resultados demostraron la viabilidad de que las personas con tetraplejia, años después de la lesión del sistema nervioso central, puedan recrear un control multidimensional útil de dispositivos complejos directamente a partir de una pequeña muestra de señales neuronales (DiLorenzo et al., 2006).

Se puede apreciar en base a sus resultados, que los estudios dentro del proyecto Braingate se ramifican en dos direcciones de aplicación en las personas con parálisis: respecto al manejo de un mouse de computador y respecto al manejo de prótesis robóticas, específicamente de las extremidades superiores. Además, este esfuerzo investigativo, que se reconoce según lo observado en esta 
revisión como el de mayor presencia en cuanto a la entrega de evidencia científica, tiene totalmente direccionada su proyección hacia el uso de una interfaz de tipo invasiva. A diferencia de esto, se observó que estudios donde se busca habilitar a personas con hemiplejia prefieren el uso de técnicas no invasivas, no obstante dichos estudios no han tenido una evidencia e impacto correspondiente tan relevante como el del proyecto Braingate, constituyendo esfuerzos más aislados pero que avizoran un posible campo de desarrollo dirigido específicamente a personas con secuelas posteriores a infartos cerebrales, que aun cuando pueden no vivenciar impedimentos físicos tan extremos como el de la cuadriplejia, podrían también optar por una mejor calidad de sobrevida, al poder contar con interfaces que faciliten su autonomía.

El auge del campo de estudio de interfaces neuronales en la última década y el éxito de diversas tecnologías en su aplicación en humanos observado en los estudios revisados, serían indicadores de que se proyectará como una subdisciplina establecida dentro de las neurociencias y también en la neuroingeniería, posibilitando la creación de neurotecnología invasiva y no invasiva para su pareamiento con el funcionamiento del sistema nervioso (Birbaumer, 2006; Hatsopoulos \& Donoghue, 2009).

Los dispositivos que se desarrollaron en décadas previas, partiendo por los más modestos en cuanto a capacidades como los que permiten el control de deletreo, potencialmente se irán extendiendo hasta posibilitar muchas actividades cotidianas que actualmente requiere el apoyo de cuidadores (Donoghue, 2008). También, como se mencionó anteriormente, se avizora que los sistemas invasivos recibirán un mayor énfasis en los años venideros, ya que poseen una serie de ventajas en cuanto a no requerir demandas atencionales sustanciales, ni aprendizaje (al menos para su uso inicial), permiten un control más natural, pudiendo ser más expandibles a señales de control múltiples y discretas. Siendo así, es probable que sigan surgiendo ensayos en humanos de diversos dispositivos en fase piloto. La automatización, miniaturización, y desarrollo de sistemas inalámbricos completamente implantables es probable que sea logrado en el corto plazo a través de avances de la ingeniería, mejores estrategias de decodificación y control adaptativo, y una comprensión mayor de las señales subyacentes (Lee, Liu, \& Apuzzo, 2013).
Cabe señalar que respecto a los métodos invasivos (sensores directos) se han levantado algunos cuestionamientos en cuanto a si efectivamente proveen señales suficientemente estables y confiables como para asumir el riesgo de aceptar un implante neuroquirúrgico y la presencia a largo plazo de un cuerpo extraño en la corteza. En base a esto se han presentado argumentos a favor del uso exclusivo de métodos indirectos con sistemas basados en EEG. No obstante, dichos cuestionamientos no han tomado en cuenta el hecho de que ya existe permiso por parte del departamento de control de alimentos y medicamentos de Estados Unidos (Food and Drug Administration, [FDA]) para el uso de implantes neurológicos en humanos, con una baja incidencia de complicaciones asociadas a su implementación. Por citar un ejemplo, Donoghue (2008) menciona que alrededor de 30.000 personas que sufren la enfermedad de Parkinson ya han recibido tratamiento de estimulación cerebral profunda, para lo cual se les ha implantado electrodos varios centímetros hacia el interior del cerebro de modo tal de entregar estimulación al núcleo subtalámico para reducir los síntomas de Parkinson. Por lo tanto, implantar sensores en la superficie del cerebro (como en el caso de las interfaces neuronales directas) no parecería presentar riesgos mas allá de los que presentan otros dispositivos de implante.

A su vez las preocupaciones respecto al aspecto ético asociado al conocer procesos neuronales internos referentes a intenciones, pueden ser respondidas en cuanto a que la elección de aceptar esta tecnología como parte de ensayos de investigación esta determinada por el potencial usuario, después de un largo y cuidadoso proceso de entrega de consentimiento informado.

\section{Conclusiones}

Se puede concluir que los estudios de la última década en sistemas de interfaz neuronal aplicados en personas con parálisis constituye un cuerpo de conocimientos multidisciplinarios. Existe una mayor preferencia por un paradigma de bucle cerrado para el desarrollo de sistemas adaptativos y una proyección primordialmente hacia el desarrollo en el uso de interfaces invasivas (implantes corticales) en la operación de neuroprótesis y computadores. No obstante, también se observa el uso de técnicas no invasi- 
vas, aún cuando de manera menos prevalente y en áreas específicas asociadas al estudio de su aplicación en pacientes con hemiplejia, siendo esta también una interesante área de estudio para futuras investigaciones.

No obstante su innegable aporte clínico para mejorar la calidad de vida de personas con severa discapacidad, también es posible reconocer que esta área de trabajo investigativo ha entregado un aporte específico para el estudio y el desarrollo de conocimiento a las neurociencias. Es destacable en dicho sentido, el que ha ofrecido un marco diferenciado para el estudio de problemas científicos básicos acerca de la codificación, representación, y plasticidad en ensambles neuronales. Así mismo y de acuerdo a los desafíos existentes en cuanto a la optimización de los dispositivos, sería esperable que junto a todo lo anterior, esta área de estudio brinde en el futuro una mayor comprensión acerca de cómo los sistemas de neuronas computan, se adaptan e interactúan con el mundo externo (Hatsopoulos \& Donoghue, 2009).

\section{Referencias}

Birbaumer, N. (2006). Breaking the silence: brain-computer interfaces $(\mathrm{BCl})$ for communication and motor control. Psychophysiology, 43(6), 517-532. doi:10.1111/ j.1469-8986.2006.00456.x

Bundy, D. T., Wronkiewicz, M., Sharma, M., Moran, D. W., Corbetta, M., \& Leuthardt, E. C. (2012). Using ipsilateral motor signals in the unaffected cerebral hemisphere as a signal platform for brain-computer interfaces in hemiplegic stroke survivors. J Neural Eng, 9(3), 036011. doi: 10.1088/1741-2560/9/3/036011

Caria, A., Weber, C., Brotz, D., Ramos, A., Ticini, L. F., Gharabaghi, A., . . B Birbaumer, N. (2011). Chronic stroke recovery after combined $\mathrm{BCl}$ training and physiotherapy: a case report. Psychophysiology, 48(4), 578-582. doi: 10.1111/j.1469-8986.2010.01117.x

Carmena, J. M., Lebedev, M. A., Crist, R. E., O'Doherty, J. E., Santucci, D. M., Dimitrov, D. F.,... Nicolelis MA. (2003). Learning to control a brain-machine interface for reaching and grasping by primates. PLoS Biology, 1(2), E42-E42. doi:10.1371/journal.pbio.0000042

Chadwick, E. K., Blana, D., Simeral, J. D., Lambrecht, J., Kim, S. P., Cornwell, A. S., . . Kirsch, R. F. (2011). Continuous neuronal ensemble control of simulated arm reaching by a human with tetraplegia. J Neural Eng, 8(3), 034003. doi: 10.1088/1741-2560/8/3/034003

Chapin, J. K. (2000). Neural prosthetic devices for quadriplegia. Current Opinion in Neurology, 13(6), 671.

DiLorenzo, D. J., Walker, C. F., Davis, R., \& Gross, R. E. (2006). History and Overview of Neural Engineering, en Biomedical Engineering Fundamentals (pp. 29-2129-19): CRC Press.
Dobkin, B. H. B. (2007). Brain-computer interface technology as a tool to augment plasticity and outcomes for neurological rehabilitation. The Journal of physiology, 579(Pt 3), 637-642. doi:10.1113/jphysiol.2006.123067

Donoghue, J. P. (2008). Bridging the brain to the world: a perspective on neural interface systems. Neuron, 60(3), 511-521. doi:10.1016/j.neuron.2008.10.037

Donoghue, J. P., Hochberg, L. R., Nurmikko, A. V., Black, M. J., Simeral, J. D., \& Friehs, G. (2007a). Neuromotor prosthesis development. Medicine and health, Rhode Island, 90(1), 12-15.

Donoghue, J. P., Nurmikko, A., Black, M., \& Hochberg, L. R. (2007b). Assistive technology and robotic control using motor cortex ensemble-based neural interface systems in humans with tetraplegia. The Journal of physiology, 579(Pt 3), 603-611. doi:10.1113/jphysiol.2006.127209

Donoghue, J. P., Suner, S., \& Sanes, J. N. (1990). Dynamic organization of primary motor cortex output to target muscles in adult rats. II. Rapid reorganization following motor nerve lesions. Experimental brain research. Experimentelle Hirnforschung. Expérimentation cérébrale, 79(3), 492-503.

Enzinger, C., Ropele, S., Fazekas,F., Loitfelder, M., Gorani, F., Seifert, T.,... Müller-Putz G. (2008). Brain motor system function in a patient with complete spinal cord injury following extensive brain-computer interface training. Audio, Transactions of the IRE Professional Group on, 190(2), 215-223. doi:10.1007/s00221-008-1465-y

Evarts, E. V. (1968). Relation of pyramidal tract activity to force exerted during voluntary movement. Journal of neurophysiology, 31(1), 14-27.

Fetz, E. E. (1969). Operant conditioning of cortical unit activity. Science (New York, N.Y.), 163(3870), 955-958. doi:10.1126/science.163.3870.955

Georgopoulos, A. P. A., Kalaska, J. F. J., Caminiti, R. R., \& Massey, J. T. J. (1982). On the relations between the direction of two-dimensional arm movements and cell discharge in primate motor cortex. Journal of Neuroscience, 2(11), 1527-1537.

Georgopoulos, A., Kettner, R., \& Schwartz, A. (1988). Primate motor cortex and free arm movements to visual targets in three- dimensional space. II. Coding of the direction of movement by a neuronal population. Journal of Neuroscience, 8(8), 2928-2937.

Hatsopoulos, N. G., \& Donoghue, J. P. (2009). The science of neural interface systems. Annu Rev Neurosci, 32, 249-266. doi:10.1146/annurev.neuro.051508.135241

Hochberg, L. R., Bacher, D., Jarosiewicz, B., Masse, N. Y., Simeral, J. D., Vogel, J.,... Donoghue, J. P. (2012). Reach and grasp by people with tetraplegia using a neurally controlled robotic arm. Nature, 485(7398), 372-375. Nature Publishing Group. doi:10.1038/ nature11076

Hochberg, L. R., Serruya, M. D., Friehs, G. M., Mukand, J. A., Saleh, M., Caplan, A. H.,... Donoghue, J. P. (2006). Neuronal ensemble control of prosthetic devices by a human with tetraplegia. Nature, 442(7099), 164-171. doi:10.1038/nature04970

Humphrey, D. R. D., Schmidt, E. M. E., \& Thompson, W. D. W. (1970). Predicting measures of motor performance from multiple cortical spike trains. Science (New York, N.Y.), 170(959), 758-762. doi:10.1126/ science.170.3959.758 
Kennedy, P. R. P., \& Bakay, R. A. R. (1998). Restoration of neural output from a paralyzed patient by a direct brain connection. Neuroreport, 9(8), 1707-1711.

Kim, S. P., Simeral, J. D., Hochberg, L. R., Donoghue, J. P., Friehs, G. M., y Black, M. J. (2011). Point-and-click cursor control with an intracortical neural interface system by humans with tetraplegia. IEEE Trans Neural Syst Rehabil Eng, 19(2), 193-203. doi: 10.1109/ tnsre.2011.2107750

Kim, S.-P., Simeral, J. D., Hochberg, L. R., Donoghue, J. P., \& Black, M. J. (2008). Neural control of computer cursor velocity by decoding motor cortical spiking activity in humans with tetraplegia. Journal of neural engineering, 5(4), 455-476.

Kugler, J. J. (1991). Electroencephalography 60 years later. Recenti progressi in medicina, 82(3), 163-165.

Kübler, A., Nijboer, F., Mellinger, J., Vaughan, T. M., Pawelzik, H., Schalk, G.,... Wolpaw, M. D. (2005). Patients with ALS can use sensorimotor rhythms to operate a brain-computer interface. Neurology, 64(10), 17751777. doi:10.1212/01.WNL.0000158616.43002.6D

Lee, B., Liu, C. Y., \& Apuzzo, M. L. (2013). A primer on brainmachine interfaces, concepts, and technology: a key element in the future of functional neurorestoration. World Neurosurg, 79(3-4), 457-471. doi: 10.1016/j. wneu.2013.01.078

Lu, C. W., Patil, P. G., \& Chestek, C. A. (2012). Current challenges to the clinical translation of brain machine interface technology. Int Rev Neurobiol, 107, 137-160. doi: 10.1016/b978-0-12-404706-8.00008-5

Nijboer, F., Sellers, E. W., Mellinger, J., Jordan, M. A., Matuz, T., Furdea, A.,... Kübler, A. (2008). A P300-based brain-computer interface for people with amyotrophic lateral sclerosis. Clinical neurophysiology : official journal of the International Federation of Clinical Neurophysiology, 119(8), 1909-1916.

Perge, J. A., Homer, M. L., Malik, W. Q., Cash, S., Eskandar, E., Friehs, G.,...Hochberg, L. R. (2013). Intra-day signal instabilities affect decoding performance in an intracortical neural interface system. J Neural Eng, 10(3), 036004. doi: 10.1088/1741-2560/10/3/036004

Piccione, F., Giorgi, F., Tonin, P., Priftis, K., Giove, S., Silvoni, S.,...Beverina, F. (2006). P300-based brain computer interface: reliability and performance in healthy and paralysed participants. Clin Neurophysiol, 117(3), 531-537. doi: 10.1016/j.clinph.2005.07.024
Schwartz, A. B., Taylor, D. M., \& Tillery, S. I. (2001). Extraction algorithms for cortical control of arm prosthetics. Current Opinion in Neurobiology, 11(6), 701-707. doi:10.1016/S0959-4388(01)00272-0

Simeral, J. D., Kim, S. P., Black, M. J., Donoghue, J. P., \& Hochberg, L. R. (2011). Neural control of cursor trajectory and click by a human with tetraplegia 1000 days after implant of an intracortical microelectrode array. J Neural Eng, 8(2), 025027. doi: 10.1088/17412560/8/2/025027

Truccolo, W., Friehs, G. M., Donoghue, J. P., \& Hochberg, L. R. (2008). Primary motor cortex tuning to intended movement kinematics in humans with tetraplegia. Journal of Neuroscience, 28(5), 1163-1178. doi:10.1523/ JNEUROSCI.4415-07.2008

Urrutia, G., \& Bonfill, X. (2010). Declaración PRISMA: una propuesta para mejorar la publicación de revisiones sistemáticas y metaanálisis. Medicina clínica, 135(11), 507-511.

Velliste, M. M., Perel, S. S., Spalding, M. C. M., Whitford, A. S. A., \& Schwartz, A. B. A. (2008). Cortical control of a prosthetic arm for self-feeding. Audio, Transactions of the IRE Professional Group on, 453(7198), 1098-1101. doi:10.1038/nature06996

Walter, W. G. (1964). Slow potential waves in the human brain associated with expectancy, attention and decision. Archiv für Psychiatrie und Nervenkrankheiten Vereinigt mit Zeitschrift für die Gesamte Neurologie und Psychiatrie, 206(3), 309-322. doi:10.1007/ BF00341700

Wessberg, J., Stambaugh, C. R., Kralik, J. D., Beck, P. D., Laubach, M., Chapin, J. K.,... Nicolelis M. A. (2000). Real-time prediction of hand trajectory by ensembles of cortical neurons in primates. Nature, 408(6810), 361-365. doi:10.1038/35042582

Wolpaw, J. R., Birbaumer, N., McFarland, D. J., Pfurtscheller, G., \& Vaughan, T. M. (2002). Brain-computer interfaces for communication and control. Clinical neurophysiology : official journal of the International Federation of Clinical Neurophysiology, 113(6), 767-791.

Para citar este artículo:

Lucero, B., \& Muñoz-Quezada, M. T. (2014). Sistemas de interfaz neuronal y su desarrollo en las neurociencias: revisión bibliográfica sistemática acerca de su aplicación en personas con parálisis. Ciencias Psicológicas VIII (2): 187 - 197 